\title{
Modeling and forecasting of epidemic spreading of the SARS-CoV-2 based in the nonlinear stochastic dynamics
}

Leonardo S. Lima ( $\sim$ Islima7@yahoo.com.br)

Federal Center for Technological Education of Minas Gerais

\section{Research Article}

Keywords: Stochastic dynamics, Fokker-Planck equation

Posted Date: May 19th, 2020

DOI: https://doi.org/10.21203/rs.3.rs-29533/v1

License: (1) (1) This work is licensed under a Creative Commons Attribution 4.0 International License.

Read Full License

Version of Record: A version of this preprint was published at Scientific Reports on May 19th, 2020. See the published version at https://doi.org/10.1038/s41598-021-91024-6. 


\title{
Modeling and forecasting of epidemic spreading of the SARS-CoV-2 based in the nonlinear stochastic dynamics
}

\author{
Leonardo S. Lima \\ Physics Department, Federal Center for Technological Education of Minas Gerais, 30510-000, Belo Horizonte, MG, Brazil.
}

(Dated: May 17, 2020)

\begin{abstract}
In this paper, we propose a stochastic model based on Itô diffusion as mathematical model for time evolution of new cases $N(t)$ of the SARS-CoV-2 (COVID-19) in each day $t$. We propose a correspondent stochastic differential equation (SDE) analogs to classical differential equations for epidemic growing for some diseases as smallpox and typhoid fever. Furthermore, we made an analysis using the Fokker-Planck equation giving an estimating of the new cases in each day $t$ as the mean half-width of the distribution $P(N, t)$ of new cases. Our results display that the model based on Itô diffusion fit well to the results supported by healthy Brazilian agencies due to large uncertain in the official results and to the low number of tests realized generating so a strong randomness in the official data.
\end{abstract}

\section{INTRODUCTION}

The current outbreak of coronavirus disease (COVID-19) has become a global crisis due to its quick and wide spread over the world. A understanding of the dynamic of the disease or forecasting of the spread of it would greatly enhance the control and prevention.[1-9]

On the other hand, the use of models and mathematical methods for theoretical physicists to the study the spread of contagious diseases goes back a least to some works by Daniel Bernoulli in XVIII century on smallpox,[14] where in nowadays, many mathematical models have been proposed and studied for many different diseases.[10-13]

Some diseases as the typhoid fever and also the COVID-19 are spread largely by carriers or individuals who can transmit the disease but who exhibit no overt symptoms. Let $x$ and $y$ denote the proportions of susceptible and carriers, respectively, in the population, suppose that carriers are identified and removed from the population at a rate $\alpha$, so that $d y / d t=-\alpha y$. Suppose besides, that the disease spreads at a rate proportional to the product of $x$ and $y$, thus $d x / d t=-\gamma x y$. We can determine by solution of the set of equations $y$ at any time $t$ subject to initial condition $y(0)=y_{0}$ and find the proportion of the population that escapes of the epidemic by finding the limiting value of $x$ as $t \rightarrow \infty$ from the initial condition $x(0)=x_{0}$. For diseases as the smallpox that, once contracted and survived, confers a lifetime immunity, considering the cohort of individuals born in a given year and let $n(t)$ be the number of these individuals surviving a time $t$ later. Let $x(t)$ be the number of members of this cohort who have not smallpox by year $t$ and who are therefore still susceptible. Let $\alpha$ be the rate at which susceptible contract smallpox, and let $\nu$ be the rate at which people who contract smallpox die from the disease. Finally, let $\mu(t)$ be the death rate from all cases other than smallpox. Then $d x / d t$, the rate at which the number of susceptible declines, is given by $d x / d t=-[\alpha+\mu(t)] x$, where the first term of the right side is the rate at which susceptible contract smallpox, and the second term is the rate at which they die from all other cases. Also $d n / d t=-\nu \alpha x-\mu(t) n$, where $d n / d t$ is the death rate, and the two terms on the right side are the death rates due to all other causes, respectively. We make $z=x / n$ and obtain that $z$ satisfies the equation $d z / d t=-\alpha z(1-\nu z), z(0)=1$ that does not depend on $\mu(t)$. Bernoulli estimated that $\nu=\alpha=1 / 8$. Using these values, we can determine the proportion of individuals who have not had smallpox yet.

If we allow for some randomness in some coefficients of the differential equation, we often obtain a more realistic mathematical model of the situation where the size of the population at time $t$ and $\alpha(t)$ being the relative rate of growth at time $t$. It might happen that $\alpha(t)$ is not completely known, but subject to some random environmental effects, so that we have $\alpha(t)=r(t)+$ "noise", where we do not know the exact behaviour of the noise term, only its probability distribution. The function $r(t)$ is assumed to be non random.[15-19]

In this paper, we investigate the Itô diffusion model with additive white noise and nonlinear terms as a possible model for the spread of the COVID-19 in Brazil. The employment of this type of analysis already has been many employed for us in the study of the price dynamics of the financial market.[20-24] Due to uncertainly in the official data about the real cases numbers generated by the low number of tests made in the population generates a large randomness in the data and therefore, makes the use of the stochastic analysis greatly adequate to treat the spread of time evolution of the COVID-19. The plan of this paper is the following. In section II, we describe the stochastic model. In section III, we present the analytical results by Fokker-Planck equation. In section IV, we present our conclusions and final remarks. 

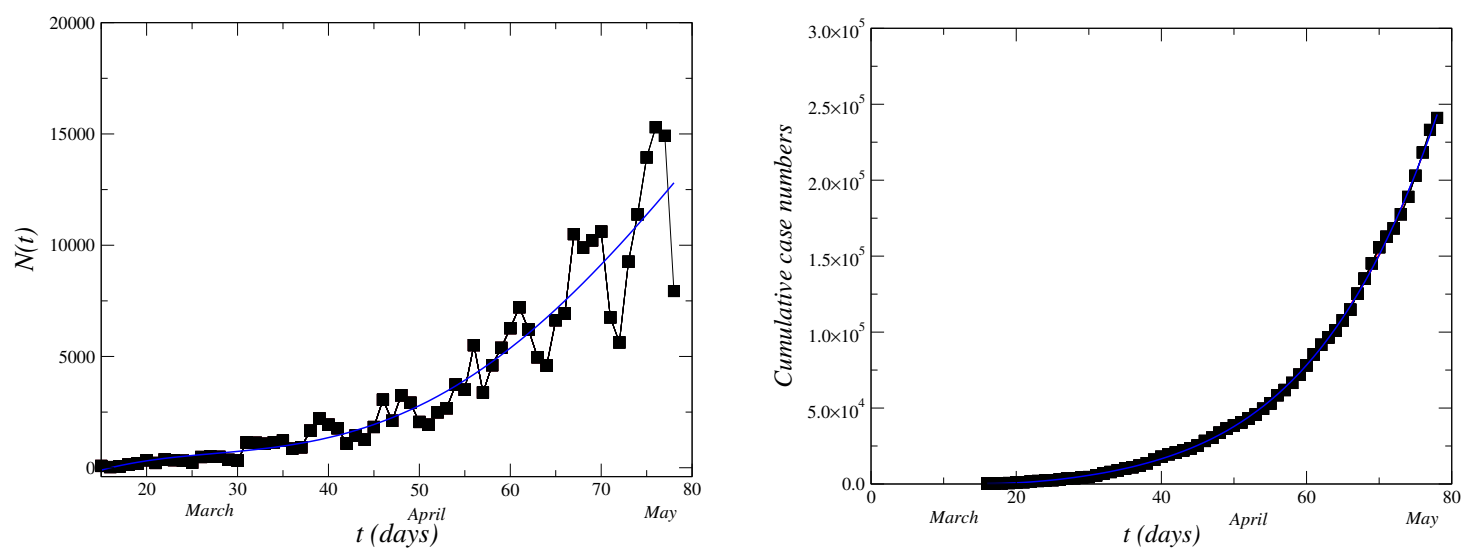

FIG. 1. Time evolution of new cases $N(t)$ in Brazil. The data was supported by the Brazilian ministry of health. The behavior zig-zag in the results is reflected by the noisy stochastic term in Eq. (1). The solid-red-line in the figure is a Fit of least-squares on results $(f(t)$ term) in Eq. (1). On the right side we display the cumulative total case number and the fit of least squares on the results.

\section{MODEL}

The behavior of the new cases number $N(t)$ of infected by coronavirus registered in the Brazil as function of time (days) and the total cumulative cases number registered since 15th March, 2020 is displayed in Fig. 1. From fit of least minimums squares on the set of officials data of the Brazilian ministry of health, we estimate the curve for the behavior of the new cases of COVID-19 given by a polynomial of fourth order of $t$ (solid-red-line) in the Fig. 1 given as $N(t)=-3976.7+459.85 t-17.384 t^{2}+0.28084 t^{3}-0.0012588 t^{4}$. The data are by May $15^{\text {th }}$. The zig-zag behavior in the range of large $t$ reflects in an increase of the uncertainty in the data and the population isolation conditions. For modeling of this behavior, we add a random term together with nonlinear terms in Eq. (1) with aim to simulate the effect this uncertainty. The $\alpha$ and $\nu$ parameters and the nonlinear polynomial added comes from the Bernoulli model for the spreading of the smallpox with the value $\alpha=\nu=1 / 8$ obtained by him. As the COVID-19 as the smallpox, once contracted and survived, confers a lifetime immunity, considering a group of individuals in a given time $t$ where we have a number of these individuals surviving a time $t$ later, we propose then that the model for the new cases in each day $t$ obeys the following stochastic differential equation in Itô calculation.

$$
d N(t)=[f(t)+A(N(t), t)] d t+B(N(t), t) \circ d W
$$

where $f(t)$ is a polynomial of $n$ degree $(n=4)$. Furthermore, we have an increase in the inclination of the curve since March $30^{\text {th }}$ which may be the effect of quarantine measures adopted. In the equation above, $W(t)$ is the Winner process or Brownian motion. Furthermore, we assume that $W(t)$ is a Markovian process with normal distribution. The equation above can be used to describe the behavior of a particle in Brownian motion under action of an polynomial potential of $n$ degree.[25] Moreover, we have a dissipative force given by $-\gamma \dot{x}$ represented by the friction term in the Langevin's equation and an environment stochastic white noise $\zeta(t)$, which if relates with the Winner process $W(t)$ by $W(t)=\int_{t_{0}}^{t} \zeta\left(t^{\prime}\right) d t^{\prime}$. Although $W(t)$ be the integral of $\zeta(t)$, the inverse is not true, i.e. $\zeta(t) \neq d W(t) / d t$, since $\frac{d W(t)}{d t}=\lim _{\Delta t \rightarrow 0} \frac{\Delta W(\Delta t)}{\Delta t} \sim \frac{1}{\sqrt{\Delta t}} \rightarrow \infty$.

\section{RESULTS}

We perform the simulation of the model Eq. (1) with the term $\beta W(t)$ being the Winner increment and an additive white noise of standard deviation $\sigma_{w}=\sqrt{\Delta t}$. We can write the Winner increment as $\beta d W(t) \sim \sqrt{d t} \beta R_{G}$, where $R_{G}$ is an aleatory generator number with a Gaussian distribution of mean zero and variance $\sigma_{w}^{2}=1$. We have the time series of the change of new cases number oscillating quickly within as displayed in Fig. 1 of data supported in the period considerate.

From the stochastic equation Eq. (1), we obtain the time development of an arbitrary function $f(X(t))$ by using of the Itô formula

$$
f[X(t)+d X(t)]-f[X(t)]=\partial_{x} f[X(t)]\left\{\left[f\left(t^{\prime}\right)-\alpha X\left(t^{\prime}\right)\left(1-\nu X\left(t^{\prime}\right)\right)\right] d t+\beta d W\right\}+\frac{\beta^{2}}{2} \partial_{x}^{2} f[X(t)](d W)^{2},
$$


where higher order terms have been discarded, and $(d W(t))^{2}=d t$. Taking the average of both sides in the equation above and defining $\gamma=\beta^{2}$, we obtain

$$
\left\langle\frac{\partial f}{\partial t}\right\rangle=\left\langle\left[\frac{\partial f}{\partial x}\{[f(t)-\alpha x(1-\nu x)] d t+\beta d W\}+\frac{\gamma}{2} \frac{\partial^{2} f}{\partial x^{2}}\right]\right\rangle .
$$

Using

$$
\begin{array}{r}
\frac{d}{d t}\langle f(X(t))\rangle=\frac{d}{d t} \int_{-\infty}^{\infty} d x f(x) P(x, t)=\int_{-\infty}^{\infty} d x f(x) \frac{\partial}{\partial t}[P(x, t)]=\int_{-\infty}^{\infty} \frac{\partial f}{\partial x}\{[f(t)-\alpha x(1-\nu x)]\} P(x, t) d x \\
+\frac{\gamma}{2} \int_{-\infty}^{\infty} \frac{\partial^{2} f}{\partial x^{2}} P(x, t) d x
\end{array}
$$

we integrate by parts and discard surface terms to obtain

$$
\int_{-\infty}^{\infty} d x f(x) \frac{\partial}{\partial t}[P(x, t)]=\int_{-\infty}^{\infty} f(x) \frac{\partial}{\partial x}\{[f(t)-\alpha x(1-\nu x)] P(x, t)\} d x+\frac{\gamma}{2} \int_{-\infty}^{\infty} f(x) \frac{\partial^{2}}{\partial x^{2}}[P(x, t)] d x .
$$

and hence

$$
\frac{\partial}{\partial t} P(x, t)=-\frac{\partial}{\partial x}\{[f(t)-\alpha x(1-\nu x)] P(x, t)\}+\frac{\gamma}{2} \frac{\partial^{2}}{\partial x^{2}} P(x, t) .
$$

The associated Fokker-Planck equation to the above model is given by

$$
\frac{\partial P(x, t)}{\partial t}=\frac{\partial}{\partial x}\{[f(t)-\alpha x(1-\nu x)] P(x, t)\}+\frac{\gamma}{2} \frac{\partial^{2}[P(x, t)]}{\partial x^{2}} .
$$

taking the Fourier transform of the Fokker-Planck equation, we can simultaneously guarantee the normalization of the probability density in which $P(x)$ is reasonably well behaved. We take the boundaries at infinity for $P(x, t)$ as $\lim _{x \rightarrow \infty} P(x, t)=0$ and therefore $\partial_{x} P(x)$ being reasonably well behaved. As $\lim _{x \rightarrow \infty} \partial_{x} P(x, t)=0$ thus, a nonzero current of probability at infinity will usually require that the terms in the equation above become infinite there[16]. We use the initial condition $P\left(x=x_{0}, t=0\right)=P_{0}$. For solving the Fokker-Plank equation above independent on time we make the power series expansion $P(x, t)=\sum_{n=0}^{\infty} a_{n}(t) x^{n}$ to obtain

$$
\begin{array}{r}
\frac{\partial P}{\partial t}=\alpha(1-2 \nu x) P-[f(t)-\alpha x(1-\nu x)] \frac{\partial P}{\partial x}+\frac{\gamma}{2} \frac{\partial^{2} P}{\partial x^{2}} \\
\sum_{n=0}^{\infty}\left(\frac{d a_{n}}{d t}+f(t)(n+1) a_{n+1}\right) x^{n}=[\alpha(1-\nu x)-\alpha \nu x] \sum_{n=0}^{\infty} a_{n} x^{n}+\alpha x(1-\nu x) \sum_{n=0}^{\infty} n a_{n} x^{n-1}+\frac{\gamma}{2} \sum_{n=0}^{\infty} n(n-1) a_{n} x^{n-2} .
\end{array}
$$

We obtain the relations

$$
\alpha \sum_{n=0}^{\infty} a_{n} x^{n}-2 \nu \alpha \sum_{n=1}^{\infty} a_{n-1} x^{n}+\sum_{n=1}^{\infty} n a_{n} x^{n}-\alpha \nu \sum_{n=2}^{\infty}(n-1) a_{n-1} x^{n}-\frac{\gamma}{2} \sum_{n=0}^{\infty}(n+1)(n+2) a_{n+2} x^{n}=k
$$

For separation constant $k=0$, one obtains the following recurrence relations

$$
\begin{array}{r}
a_{2}=-\frac{\alpha}{\gamma} a_{0} \\
a_{3}=2 \nu \alpha a_{0}-a_{1}(1+\alpha) \\
a_{4}=\frac{\alpha}{3 \gamma^{2}}\left(\frac{\alpha}{2}-1\right) a_{0}+\frac{\nu \alpha}{2 \gamma} a_{1}
\end{array}
$$

for $0 \leq m \leq 2$, and

$$
a_{m+2}=\frac{2}{\gamma} \frac{\left[\alpha \nu(m+1) a_{m-1}-(\alpha+m) a_{m}\right]}{(m+2)(m+1)},
$$




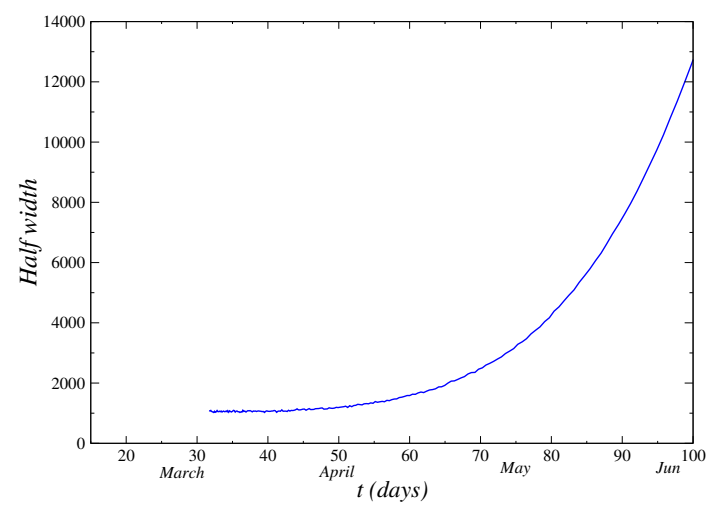

FIG. 2. Time evolution of the half width of the distribution $\sigma$ with $t$. The half width gives an estimating of the new cases in the day $t$.

for $m>2$. Additionally, we have

$$
\frac{d a_{n}(t)}{d t}+f(t)(n+1) a_{n+1}(t)=k .
$$

Therefore, we obtain $P(x)$ in the form

$$
\begin{aligned}
P(x)=\left\{1-\frac{\alpha}{\gamma} x^{2}+2 \nu \alpha x^{3}+\right. & \left.\frac{\alpha}{3 \gamma^{2}}\left(\frac{\alpha}{2}-1\right) x^{4}-\frac{1}{10 \gamma}\left[2 \nu(3+\alpha)+\frac{2 \nu \alpha}{\gamma}(\nu+\alpha)\right] x^{5} \cdots\right\} a_{0} \\
& +\left\{x-(1+\alpha) x^{3}+\frac{\nu \alpha}{2 \gamma} x^{4}+\frac{1}{10 \gamma}(3+\alpha)(1+\alpha) x^{5}+\cdots\right\} a_{1} .
\end{aligned}
$$

where the constants $a_{0}$ and $a_{1}$ are determined by the initial conditions $P(0,0)=P_{0}$ and $\partial_{x} P(x, 0)=0$ em $x=0$. We obtain $a_{0}=P_{0}$ and $a_{1}=0$, being $k$ the separation constant in Eq. (7). From the normalization condition, the second term in the density probability above must be zero and therefore, all coefficients $a_{1}$ must cancel. Therefore, we have

$$
P(x, t)=P_{0}\left\{1-\frac{\alpha}{\gamma} x^{2}+2 \nu \alpha x^{3}+\frac{\alpha}{3 \gamma^{2}}\left(\frac{\alpha}{2}-1\right) x^{4}-\frac{1}{10 \gamma}\left[2 \nu(3+\alpha)+\frac{2 \nu \alpha}{\gamma}(\nu+\alpha)\right] x^{5} \cdots\right\}
$$

To ensure the normalization of the probability density, $P_{0}$ must be non zero only within interval $-\varepsilon \leq x \leq \varepsilon$ and zero out it. Furthermore, for nonzero $k$ values we have $a_{2}+\alpha a_{0} / \gamma=k$ and all $a_{n}$ higher are zero. Consequently, we obtain

$$
P(t)=\frac{\gamma}{\alpha} P_{0} t\left(1-a_{2}(t)\right)\left(a_{n}(0)+\int_{0}^{t} f\left(t^{\prime}\right) a_{1}\left(t^{\prime}\right) d t^{\prime}\right) .
$$

Additionally, we can obtain the $n$-th moments $m_{n}=\left\langle N^{n}\right\rangle=\int_{-\infty}^{\infty} N^{n} P(N, t) d x$, where the mean half width of the probability distribution $\sigma=\sqrt{\left\langle N^{2}\right\rangle-\langle N\rangle^{2}}$ gives an estimating of the new cases numbers in the day $t$. In general, we may introduce $n$-th order moments $\mu_{n}=\left\langle\left(x-m_{1}\right)\right\rangle$ about the mean or central moments, where we have the following relations: $c_{1}=\mu_{1}, c_{2}=\mu_{2}, c_{3}=\mu_{3}, c_{4}=\mu_{4}-3 \mu_{2}^{2}$, where $c_{n}$ are the cumulants. Normalized measures often used, indicating a deviation from a Gaussian are the kurtosis $\lambda_{4}$, defined as $\lambda_{4}=\mu_{4} / \sigma^{4}-3$. We obtain the mean half width of the distribution as function of time as given as

$\sigma(t)=\left\{\frac{[N(t)]^{3}}{3} \frac{\gamma}{\alpha}\left(k-a_{2}(t)\right)\left(a_{n}(0)+\int_{0}^{t} f\left(t^{\prime}\right) a_{1}\left(t^{\prime}\right) d t^{\prime}\right)-\frac{[N(t)]^{4}}{4}\left[\frac{\gamma^{2}}{\alpha^{2}}\left(k-a_{2}(t)\right)\left(a_{n}(0)+\int_{0}^{t} f\left(t^{\prime}\right) a_{1}\left(t^{\prime}\right) d t^{\prime}\right)\right]^{2}\right\}^{1 / 2}$

For $d a_{n}(t) / d t=0$, we have $a_{n}(t)=c, a_{n+1}=k /(n+1)$ and $P(t)=p_{0}(1-k / 3)$, where $p_{0}=c P_{0}$. Therefore

$$
\sigma(t)=p_{0}\left(1-\frac{k}{3}\right) \sqrt{\frac{[N(t)]^{3}}{3}-\frac{[N(t)]^{4}}{4}} .
$$

In Fig. 2, we plot the mean half width as function of time $t$ (days). From probability density of new cases $P(N, t)$ obtained from solution of the Fokker-Planck equation Eq. (7), we calculate the variance of the distribution where 


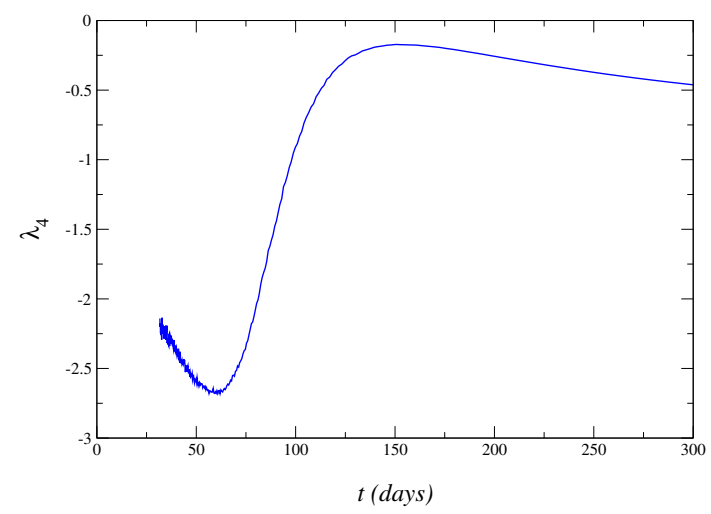

FIG. 3. Time evolution of the kurtosis $\lambda_{4}(t)$. The negative values obtained for all $t$ values gives an estimating shape of distribution that becomes nearest of a Gaussian $\lambda 4=0$ at range of large $t$ values since the firsts cases registrated in Brazil in 15 th March 2020 following the official data, by the Brazilian ministry of Health.

the standard deviation gives an estimating of the number of new cases in each day $t$. The results fit qualitatively to the official data of ministry of healthy. According of the results obtained we obtain a forecasting of increase in the new cases number in the next weeks. In Fig. 3, we display the behavior of the kurtosis (excess), $\lambda_{4}(t)$ as function of time. The excess kurtosis relates to the deviation of the tail of the distribution, as compared to Gaussian. The negative value obtained for the kurtosis for all $t$ values indicates that the shape of the distribution is next to a Wigner semicircle[18]. Furthermore, at range of $t$ where the kurtosis is nearest to zero we have the distribution nearest of a Gaussian $\left(\lambda_{4}=0\right)$ what occurs for the range of large values of $t$ as expected.

\section{CONCLUSIONS}

In a nutshell, we propose a model for the time evolution of the SARS-CoV-2 in Brazil based in the nonlinear Itô's diffusion. Our results are compared with official data supplied by the Brazilian healthy agencies where due to large uncertain in the results generated principally by the low number of tests made in the population and therefore, the underreporting generates a large uncertainly in the official results and consequently, becomes necessary, the addition of randomness in the differential equations for growing of the infected number. In a general way, a polynomial of $n$ degree in the stochastic differential equation presents local minimums and maximum beyond which the potential plummets to $-\infty$. It generates barriers separating stable from unstable regions modeling situations of crashes or crisis as for instance, the sudden break down of lock down conditions.

[1] G. Giordano, F. Blanchini, R. Bruno, P. Colaneri, A. Di Filippo, A. Di Matteo, M. Colaneri, Nat. Med. doi.org/10.1038/s41591-020-0883-7, (2020).

[2] M. Gattoa, E. Bertuzzob, L. Maria, S. Miccolid, L. Carraroe, R. Casagrandia, A. Rinaldog, PNAS 117, 10484 (2020).

[3] S. B. Bastos, D. O. Cajueiro, arXiv:2003.14288 (2020).

[4] L. S. Lima, DOI: 10.21203/rs.3.rs-24308/v1, (2020).

[5] D. Fanelli, F. Piazza, Chaos Solitons Fractals 134, 109761 (2020).

[6] S. Boccaletti, w. Ditto, G. Mindin, A. Antangana, Chaos Solitons Fractals 135, 109794 (2020).

[7] F. Ndairou, I. Area, J. J. Nieto, D. F. M. Torres, Chaos Solitons Fractals 135, 109846 (2020).

[8] Y. Zhang, C. You, Z. Cai, J. Sun, W. Hu, Xiao-Hua Zhou, doi.org/10.1101/2020.03.10.20033803 (2020).

[9] N. Crokidakis, doi.org/10.1101/2020.04.27.20081737 (2020).

[10] G. F. de Arruda, G. Petri, Y. Moreno Phys. Rev. Research 2, 023032 (2020).

[11] S. Moore, T. Rogers, Phys. Rev. Lett. 124, 068301 (2020).

[12] J. Chen, Mao-Bin Hu, M. Li, Phys. Rev. E 101, 012301 (2020).

[13] F. V. Surano, C. Bongiorno, L. Zino, M. Porfiri, A. Rizzo, Phys. Rev. E 100, 042306 (2019).

[14] W. E Boyce, R. C. DiPrima, Elementary Differential Equations 10th Edition, Wiley, United States of American (2012).

[15] Steven E. Shreve, Stochastic Calculus for Finance II Continuous-Time Models, (Springer, New York, 2004).

[16] C. Gardiner, Stochastic Methods, A Handbook for the Natural and Social Sciences, (Fourth edition, Springer, New Zealand, (2009) 
[17] B. Oksendal, Stochastic Differential Equations An Introduction with Applications, sixth edition, Springer-Verlag, Oslo (2013).

[18] P. Richmond, J. Mimkes, S. Hutzler, Econophysics and Physical Economics, Oxford, UK (2013).

[19] Kurt Jacobs, Stochastic Processes for Physicists, Cambridge University Press, New York (2013).

[20] L. S. Lima, S. C. Oliveira, Chaos, Solitons \& Fractals 136, 109792 (2020).

[21] L. S. Lima, L. L. B. Miranda, Physica A 490, 828 (2018).

[22] L. S. Lima, S. C. Oliveira, A. F. Abeilice, Pioneer Journal of Mathematics and Mathematical Sciences 23, 93 (2018).

[23] L. S. Lima, G. K. C. Santos, Physica A 512, 222 (2018).

[24] L. S. Lima, S. C. Oliveira, A. F. Abeilice, J. H. C. Melgaço, Physica A 526, 120932 (2019).

[25] J.-P. Bouchaud, R. Cont, Eur. Phys. J. B 6, 543 (1998).

\section{Declarations:}

Competing interests: I declare no competing interests. 
Figures
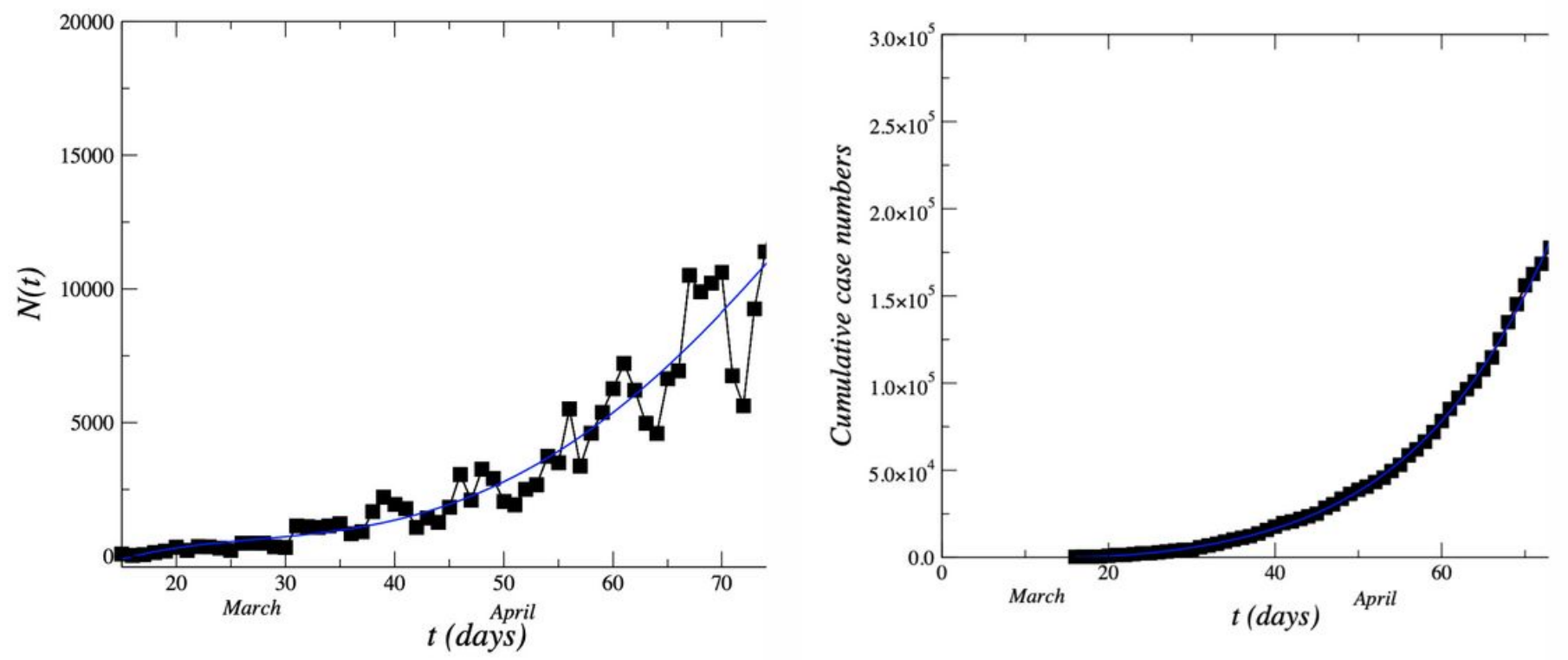

\section{Figure 1}

Time evolution of new cases $\mathrm{N}(\mathrm{t})$ in Brazil. The data was supported by the Brazilian ministry of health. The behavior zig-zag in the results is reflected by the noisy stochastic term in Eq.(1). The solid-red-line in the figure is a Fit of least-squares on results ( $f(t)$ term) in Eq.(1). On the right side we display the cumulative total case number and the fit of least squares on the results. 


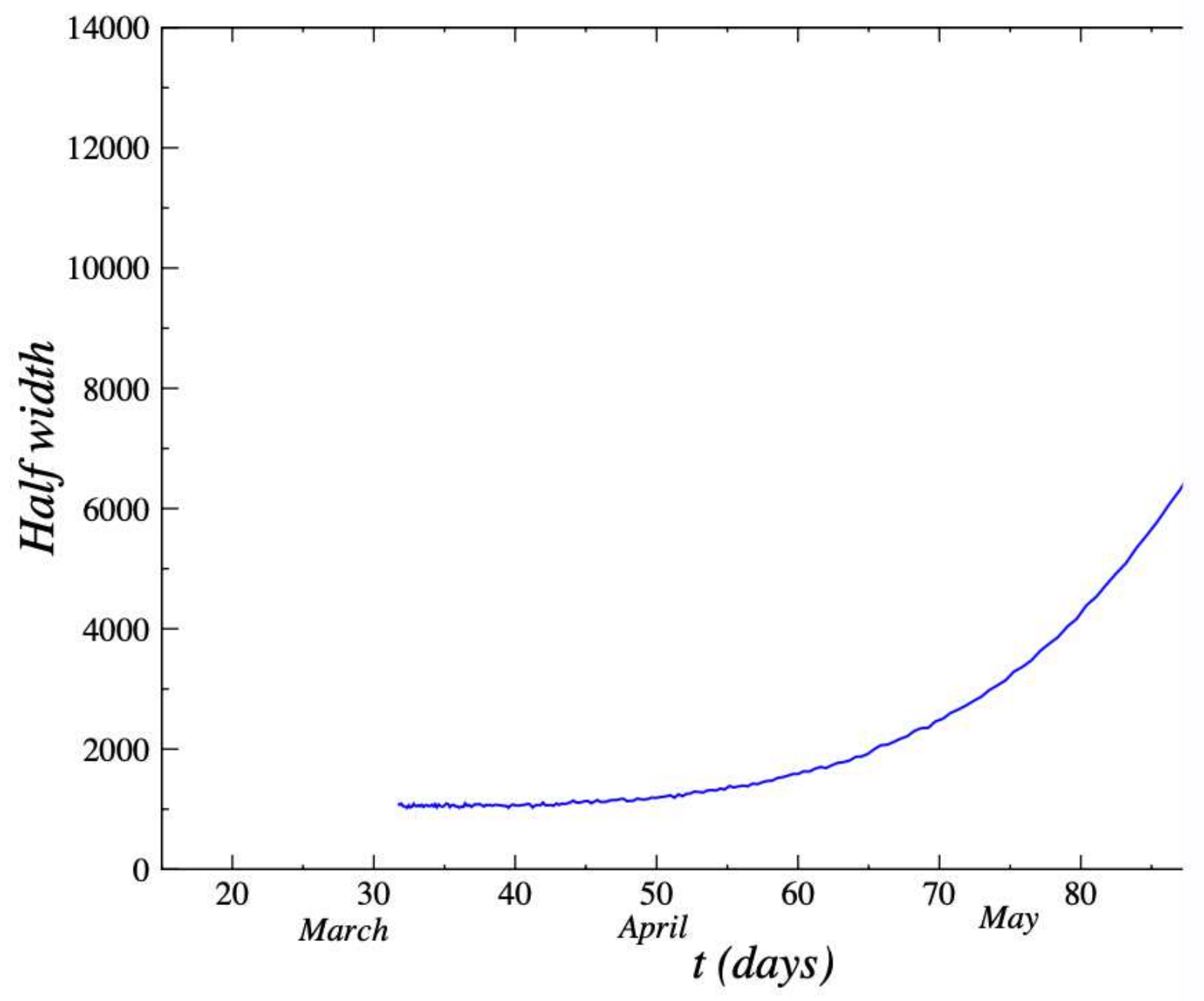

Figure 2

Time evolution of the half width of the distribution $\sigma$ with t. The half width gives an estimating of the new cases in the day $t$. 


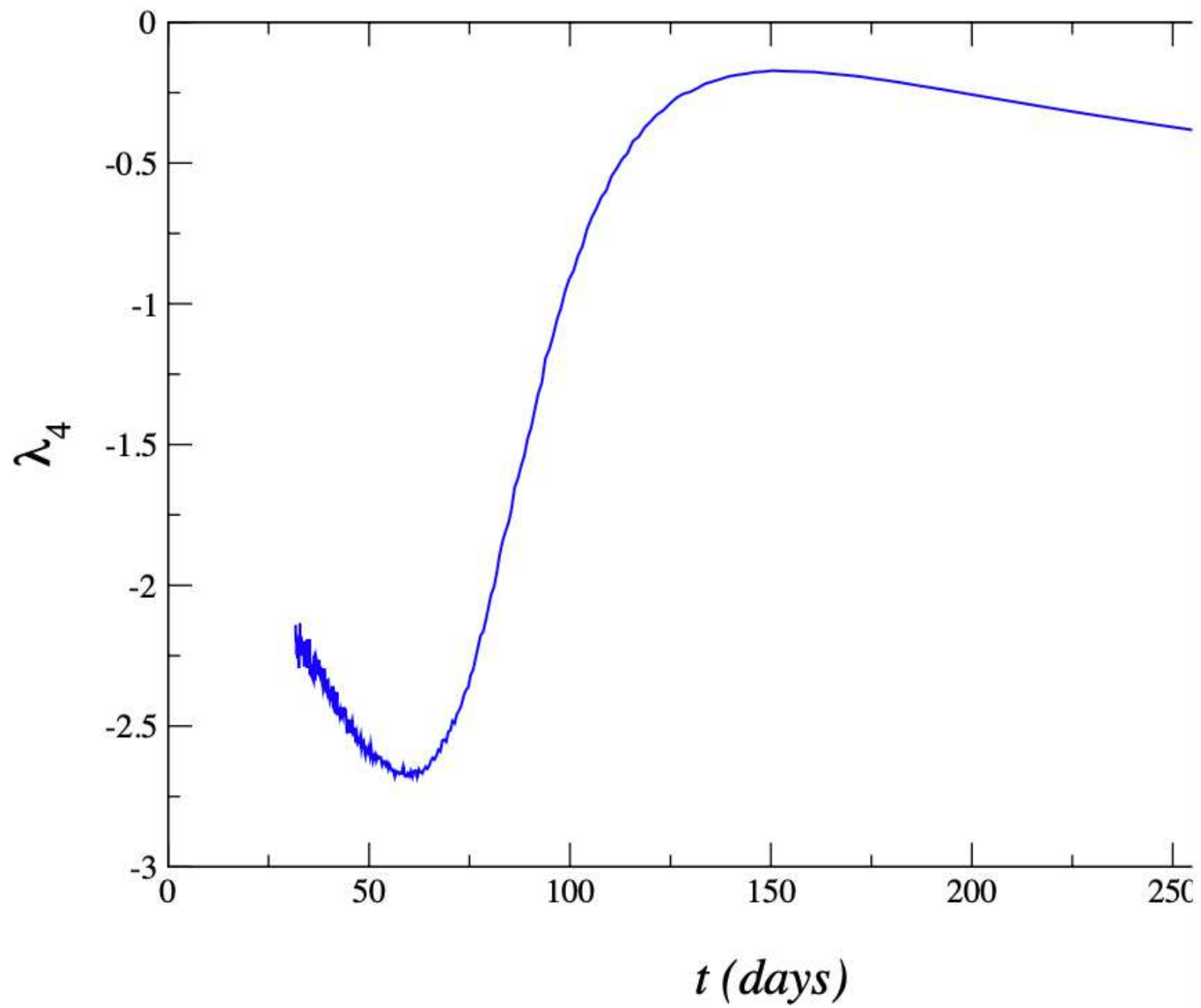

Figure 3

Time evolution of the kurtosis \$Vlambda_4(t)\$. The negative values obtained for all $t$ values gives an estimating shape of distribution that becomes nearest of a Gaussian $\$ \backslash$ lambda4 $=0 \$$ at range of large $\$$ t\$ values since the firsts cases registrated in Brazil in 15 th March 2020 following the official data, by the Brazilian ministry of Health. 